\title{
Enhanced INSURE (ENSURE): an updated and standardised reference for surfactant administration
}

\author{
Feriel Fortas ${ }^{1,2} \cdot$ Barbara Loi $^{1} \cdot$ Roberta Centorrino $^{1,2} \cdot$ Giulia Regiroli $^{1} \cdot$ Rafik Ben-Ammar $^{1}$. \\ Shivani Shankar-Aguilera ${ }^{1} \cdot$ Nadya Yousef $^{1} \cdot$ Daniele De Luca $^{1,2}{ }^{10}$
}

Received: 19 July 2021 / Revised: 5 October 2021 / Accepted: 19 October 2021 / Published online: 4 November 2021

(C) The Author(s), under exclusive licence to Springer-Verlag GmbH Germany, part of Springer Nature 2021

\begin{abstract}
There is no firm consensus about the optimal technique for the administration of exogenous surfactant in preterm neonates, and different techniques may be equally effective. The intubation-surfactant-extubation (INSURE) procedure has not been fully described, and important details, such as duration and mode of ventilation, remain unclear, leading to significant clinical practice variations and influencing its suitability and feasibility. Since the first INSURE description, our knowledge in respiratory care has largely progressed, but the technique has not been updated according to current evidence-based practice. Thus, our aim is to formally describe a modern way to perform INSURE, based on the current knowledge and technology, to increase its feasibility and patients' safety. We offer ENSURE (Enhanced INSURE) as an updated and standardised technique for surfactant administration, clarifying crucial issues of the original method by applying current state-of-the-art concepts of respiratory care. We performed a cross-sectional observational study enrolling 57 preterm neonates describing ENSURE feasibility and safety. Conclusion: ENSURE can be used as a reference technique in clinical practice, teaching and research.
\end{abstract}

\section{What is Known:}

- There is no consensus about the optimal methodfor surfactant administration. INSURE technique has been originally described many years ago without considering modern principles of neonatal respiratory care and the available state-of-the-art technology.

What is New:

- We here describe a modern way to perform INSURE, based on the current knowledge and technology. We called it ENSURE (Enhanced INSURE) and clarified crucial points of the original technique, in light of the current knowledge. We verified feasibility and safety of ENSURE in a cross-sectional observational study enrolling 57 preterm neonates.

Keywords Surfactant $\cdot$ RDS $\cdot$ Preterm $\cdot$ Neonate $\cdot$ Intubation $\cdot$ LISA

Communicated by Piet Leroy

Feriel Fortas and Barbara Loi equally contributed (shared first authorship)

Daniele De Luca

dm.deluca@icloud.com

Feriel Fortas

feriel.fortas@aphp.fr

Barbara Loi

barbara.loi@aphp.fr

Roberta Centorrino

roberta.centorrino@aphp.fr

Giulia Regiroli

giulia.regiroli@aphp.fr

Rafik Ben-Ammar

rafik.ben-ammar@aphp.fr

\section{Abbreviations \\ ENSURE Enhanced INSURE \\ ESTHER Echography-guided surfactant therapy}

Shivani Shankar-Aguilera

shankaraguilera.shivani@gmail.com

Nadya Yousef

nadya.yousef@aphp.fr

1 Division of Paediatrics and Neonatal Critical Care, "A.Béclère" Medical Centre, Paris Saclay University Hospitals, APHP, 157 rue de la Porte de Trivaux, 92140 Clamart, Paris-IDF, France

2 Physiopathology and Therapeutic Innovation Unit-INSERM U999, Paris Saclay University, Paris, France 


$\begin{array}{ll}\mathrm{EtCO}_{2} & \text { End-tidal } \mathrm{CO}_{2} \\ \text { ETT } & \text { Endotracheal tube } \\ \text { INSURE } & \text { Intubation-surfactant-extubation } \\ \text { IT } & \text { Inspiratory time } \\ \text { IV } & \text { Intravenous } \\ \text { LISA } & \text { Less invasive surfactant administration } \\ \text { LUS } & \text { Lung ultrasound score } \\ \text { NICU } & \text { Neonatal intensive care } \\ \text { NIPPV } & \text { Non-invasive positive pressure ventilation } \\ \text { PEEP } & \text { Positive end-expiratory pressure } \\ \text { RDS } & \text { Respiratory distress syndrome } \\ \text { RR } & \text { Respiratory rate } \\ \text { rSatO } & \text { Regional (cerebral) oxygen saturation } \\ \text { SatO } & \text { Peripheral oxygen saturation } \\ \text { SGA } & \text { Small for gestational age } \\ \text { VG } & \text { Volume guarantee }\end{array}$

\section{Introduction}

The intubation-surfactant-extubation (INSURE) technique and the placement of narrow catheters into the trachea (also known as "less invasive surfactant administration" (LISA)) are two common methods for surfactant administration and both have been described together in 1992 by Verder et al. [1]. After more than 30 years, there is no firm consensus about the optimal technique for the administration of exogenous surfactant in preterm neonates with respiratory distress syndrome (RDS). There is a wide variation in clinical practices [2], and different techniques could be equally effective. LISA has been widely studied and recently gained some popularity [3], but it has been criticised as not being actually less invasive than INSURE, as it may be associated with relevant side effects, lacks clear physiopathological reasons justifying alleged benefits and, finally, as LISA trials are affected by relevant biases [4, 5]. INSURE can also be considered problematic, because the technique has not been fully described, and many important details, such as duration and mode of ventilation to be applied during the procedure, remain unstandardised. Differences in a number of other factors (e.g. intubation techniques and sedation policies) result in significant clinical practice variations between centres performing INSURE [2]. Moreover, INSURE, as however practiced, should be better characterised within each NICU and clinicians should scrutinise each parameter in an effort to better align with current evidence-based practices. In fact, the lack of standardization is a concern as it prevents objective and fair comparisons amongst INSURE studies and between them and those on LISA. Also, a betterdefined technique would reduce the risk of unpredictable side effects. Thus, there is a need to standardise INSURE and align it with current state-of-the-art knowledge.
The uncertainty about the best method for surfactant administration is also undoubtedly due to several obstacles that prevent the realization of large, meaningful and welldesigned randomised controlled trials in this field. However, our knowledge in neonatal respiratory critical care has progressed and, moreover, techniques and drugs previously used only in older patients have since become available for preterm neonates. Thus, our aim is to formally describe a modern way to perform INSURE, updated to the more advanced respiratory care knowledge and technology. Although it is currently impossible to clear up all the uncertainty about surfactant administration, we hope at least to provide a reference to perform INSURE in a standardised fashion, to help teaching the technique and eventually to increase its suitability and feasibility.

\section{Enhanced INSURE (ENSURE)}

We designed an updated way to perform INSURE, and we called it: ENSURE (Enhanced INSURE - listed by the French Institute of intellectual property amongst the inventions free to be used without any copyright n.DSO2020003859, since 7 March 2020). ENSURE integrates state-of-the-art respiratory care principles and standardises crucial points from the original INSURE technique. Currently, in the context of modern neonatal critical care, INSURE presents 4 crucial issues:

1) Duration: The original INSURE did not have a clear duration, although it should be as short as possible [4, 5].

2) Monitoring: Modern monitoring to verify intubation and ensure patients' safety was not formally included in the INSURE technique and need to be considered.

3) Sedation/analgesia: Clear guidance for sedation and analgesia was not given, and too heavy or too light sedation may cause prolonged ventilation or significant discomfort and its harmful consequences, respectively.

4) Ventilatory technique: The original INSURE did not consider modern principles of gentle ventilation, which avoids the delivery of large tidal volumes and high peak pressures.

ENSURE addresses all these points considering the more recent knowledge as explained below and in Fig. 1.

\section{Duration}

ENSURE is a teamwork procedure and should be as short as possible [4,5] (Fig. 1; online video): the physician intubates the patient, while a nurse helps in the preparation and injects the sedative drugs. It is also important to have a nurse or 
an assistant timing the procedure from the confirmation of intubation to the extubation. This highlights the importance of short ventilation and raises consciousness on the amount of time passing by. Chronometers are often integrated in the neonatal intensive care (NICU) monitoring system, but a simple watch can also be used. It is important to have a short meeting before the procedure to decide the role of every participant. It is advisable to have a protocol for difficult airway management and good communication in case of emergencies [6]. As for several NICU procedures, appropriate simulation team training may be needed [6]. This latter does not need to be in high-fidelity, as our personal experience during 18 months shows that simple simulations repeated 2-3 times/year are effective [7]. With the use of timers, simulation training and good communication, ENSURE can have a maximal duration of $20^{\prime}$ (see below).

\section{Monitoring}

During ENSURE, electrocardiogram, respiratory rate and peripheral oxygen saturation (at least on a pre-ductal site) monitoring are provided. If available, cerebral saturation using near-infrared spectroscopy might also be added to estimate cerebral perfusion and oxygenation, and this may be useful to increase patients' safety, although it is not mandatory. During such a short procedure, there is usually no need to measure mean arterial pressure (unless continuous measurement through an umbilical arterial line was deemed useful and was already available), as this is known to be unrelated to actual cerebral blood flow. Furthermore, it is crucial to confirm intubation quickly and safely: this shall be done with exhaled $\mathrm{CO}_{2}$ monitoring, which is the gold standard in critical care [8]. Low-dead space, accurate exhaled $\mathrm{CO}_{2}$ detectors are commercially available and should be used to confirm the intubation also in very preterm neonates and do not need to stay in place for the whole duration of ENSURE (Fig. 1; online video). Common tube insertion formulas should be used, and the symmetric thoracic expansion can be quickly verified by auscultation or pointof-care lung ultrasound. These techniques together avoid the need for chest X-rays and unnecessary delays prolonging the procedure.

\section{Sedation/analgesia}

All surfactant administration techniques require laryngoscopy which is associated with acute cardiovascular effects, such as catecholamine release, systemic and pulmonary hypertension, tachycardia, arrhythmias and, in some cases, increased intracranial and intraocular pressures, which are particularly harmful in preterm neonates during the first week of life. The stimulation by the laryngoscope blade causes these physiologic responses since the supraglottic region is highly reflexogenic $[4,5]$. Non-pharmacological sedation and patient positioning are useful to decrease discomfort but need to be coupled with pharmacological sedation to obtain blunted physiologic effects. During ENSURE, discomfort is reduced as much as possible, albeit spontaneous breathing is preserved (or avoided only for a short period), allowing a short invasive ventilation and a quick shift to non-invasive respiratory support. During ENSURE, this is achieved by using drugs such as propofol or remifentanil. While the perfect premedication for neonatal intubation still needs to be determined, these drugs currently seem to have a favourable profile. In fact, they allow spontaneous breathing or can be antagonised, and they have quick onset and reversal. We generally prefer to use propofol, because, according to our experience and pharmacokinetic data, a low dose $(1 \mathrm{mg} / \mathrm{kg})$ [9] is usually sufficient to reach adequate sedation with a good safety (Fig. 1; online video). In fact, at least one physiological study suggests that cerebral saturation and local vascular autoregulation remain intact even if hypotension might occur [10]. Our personal experience is also consistent with these data. If arterial pressure measurements after ENSURE indicate hypotension, it is important to integrate this with the data coming from cerebral oxygenation and point-of-care echocardiography [11]. When it occurs, hypotension is usually mild and transient and needs no treatment. We do not use propofol in patients with high intrathoracic pressure, and we prefer remifentanil or ketamine. Oral intubation is usually preferred as this may allow the use of larger tubes with less leaks and seems associated with less post-extubation atelectasis [12].

\section{Ventilatory technique}

Large tidal volume and/or high peak pressure without positive end-expiratory pressure (PEEP), particularly if delivered for a long time, trigger significant lung injury in several animal models $[4,5]$. Therefore, a tidal volume of about $5 \mathrm{~mL} /$ $\mathrm{kg}$ is currently recommended with adequate PEEP of at least 5-6 $\mathrm{cmH}_{2} \mathrm{O}$ [13]. Thus, ENSURE includes a short and gentle ventilation provided with volume-targeted (i.e. volume guarantee (VG)), pressure-regulated mode, which allows clinicians to ventilate with less variable tidal volumes. This technique is available in all modern ventilators and is superior to conventional modalities without volume targeting [14]. Surfactant is injected directly into the endotracheal tube without inserting a feeding catheter into it (Fig. 1; online video). VG operates real-time pressure weaning while lung compliance quickly improves thanks to surfactant. Bag ventilation must be strictly avoided if not applied with devices providing PEEP and in-line control of delivered volume and pressure. Immediately after extubation, very preterm neonates are switched to non-invasive positive pressure ventilation (NIPPV) as this technique allows the delivery of 


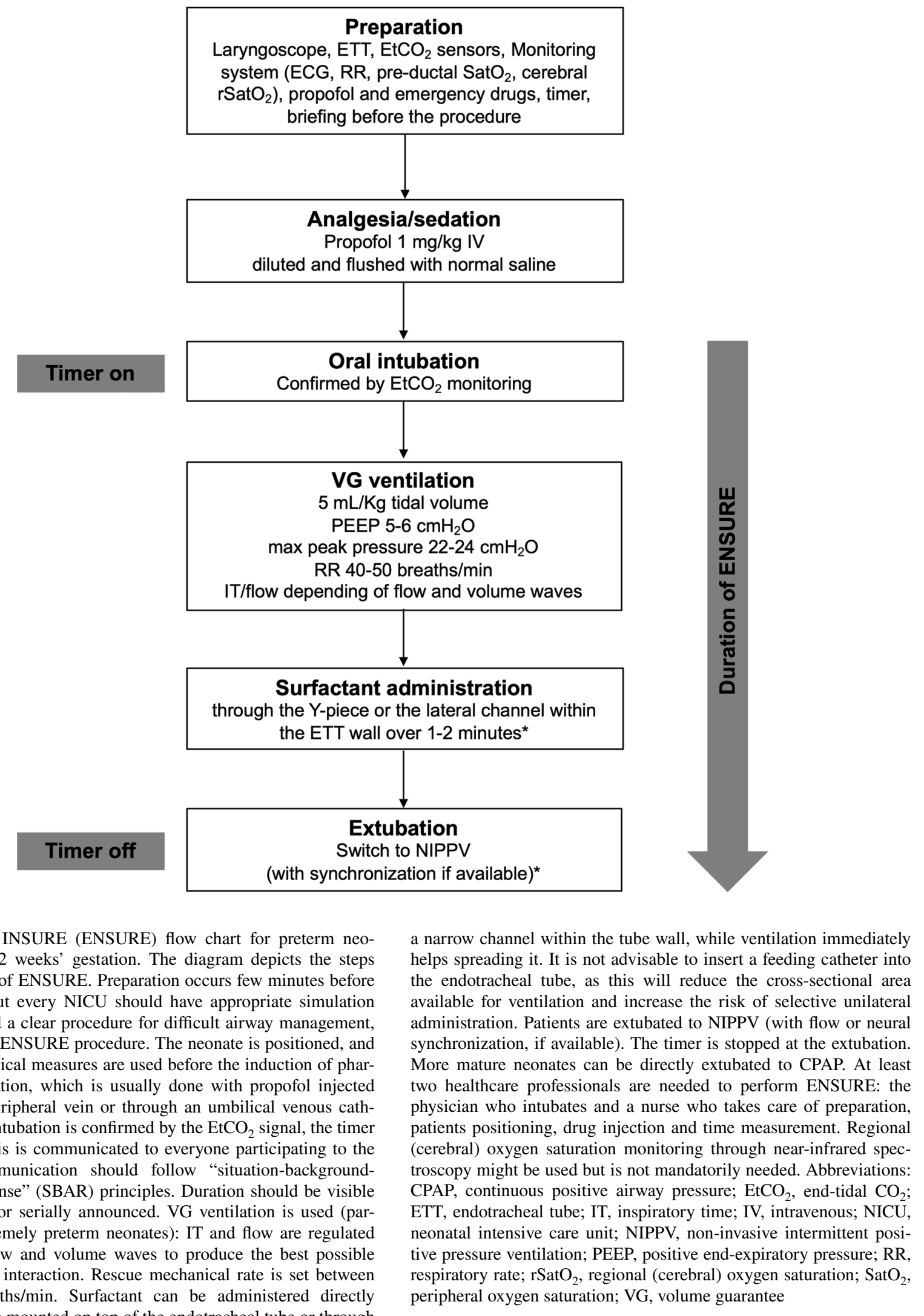

Fig. 1 Enhanced INSURE (ENSURE) flow chart for preterm neonates born at $\leq 32$ weeks' gestation. The diagram depicts the steps and peculiarities of ENSURE. Preparation occurs few minutes before the procedure, but every NICU should have appropriate simulation team training and a clear procedure for difficult airway management, independently of ENSURE procedure. The neonate is positioned, and non-pharmacological measures are used before the induction of pharmacological sedation, which is usually done with propofol injected either through peripheral vein or through an umbilical venous catheter. When oral intubation is confirmed by the $\mathrm{EtCO}_{2}$ signal, the timer is started, and this is communicated to everyone participating to the procedure. Communication should follow "situation-backgroundassessment-response" (SBAR) principles. Duration should be visible to everyone and/or serially announced. VG ventilation is used (particularly for extremely preterm neonates): IT and flow are regulated by looking at flow and volume waves to produce the best possible patient-ventilator interaction. Rescue mechanical rate is set between 40 and 50 breaths $/ \mathrm{min}$. Surfactant can be administered directly through a Y-piece mounted on top of the endotracheal tube or through a narrow channel within the tube wall, while ventilation immediately helps spreading it. It is not advisable to insert a feeding catheter into endotracheal tube, as this will reduce the cross-sectional area synchronization, if available). The timer is stopped at the extubation. More mature neonates can be directly extubated to CPAP. At least two healthcare professionals are needed to perform ENSURE: the physician who intubates and a nurse who takes care of preparation, patients positioning, drug injection and time measurement. Regional (cerebral) oxygen saturation monitoring through near-infrared spectroscopy might be used but is not mandatorily needed. Abbreviations: CPAP, continuous positive airway pressure; $\mathrm{EtCO}_{2}$, end-tidal $\mathrm{CO}_{2}$; , endracheal tube; IT, inspiratory time; IV, intravenous; NICU, peripheral oxygen saturation; $\mathrm{VG}$, volume guarantee 
higher mean airway pressure, facilitating surfactant spread and reducing extubation failure [15]. If available, flow or neural synchronization are also used, since they can improve patient-ventilator synchrony and ventilation efficiency, as compared to unsynchronised NIPPV. More mature neonates can be extubated on continuous positive airway pressure as they have a lower risk of extubation failure.

\section{Methods}

The aforementioned details allowed us to standardise the technique and implement it in our protocols since 2019. We aimed to describe an illustrative cohort of patients treated with ENSURE to verify its feasibility. Thus, a cross-sectional observational study, performed on several weeks during the first half of 2021, surveyed the ENSURE data of 57 preterm neonates. The study protocol was approved by local ethical committee (SRLF-n.21/33), and informed consent was obtained from parents upon admission. Data distribution was analysed with Shapiro-Wilk test, and descriptive statistics was applied. Data were expressed with number (\%), mean (standard deviation), or median (25th-75th percentile), according to their distribution.

\section{Experience with ENSURE}

Basic data of the analysed population are shown in Table 1; no problem was noticed during ENSURE procedure. An example of ENSURE can be seen in real life in the online supplementary video (recorded with specific parental consent). Results show that the median duration of the procedure was $8^{\prime}$ [interquartile range: $5-12^{\prime}$ ] with a minimum of $2^{\prime}$ and a maximum of $20^{\prime}$ (Fig. 2). During these periods, VG ventilation was provided with 5-6 $\mathrm{cmH}_{2} \mathrm{O}$ of PEEP and $5 \mathrm{~mL} /$ $\mathrm{kg}$ tidal volume. Significant lung injury has been triggered in animal models only using zero end-expiratory pressure

Table 1 Basic population details of neonates treated with enhanced INSURE (ENSURE). Data are expressed as mean (standard deviation), median [25th-75th percentile], or number (\%). Apgar and LUS are dimensionless scores. Abbreviations: LUS, lung ultrasound score; SGA, small for gestational age

\begin{tabular}{|c|c|}
\hline Neonates & 57 \\
\hline Gestational age (weeks) & $29(2.9)$ \\
\hline Birth weight (grams) & $1349(593)$ \\
\hline SGA neonates & $1(1.8 \%)$ \\
\hline Caesarean section & $37(64.9 \%)$ \\
\hline 5' Apgar score & $9[8-10]$ \\
\hline Male sex & $29(50.9 \%)$ \\
\hline LUS & 10 [10-12] \\
\hline
\end{tabular}

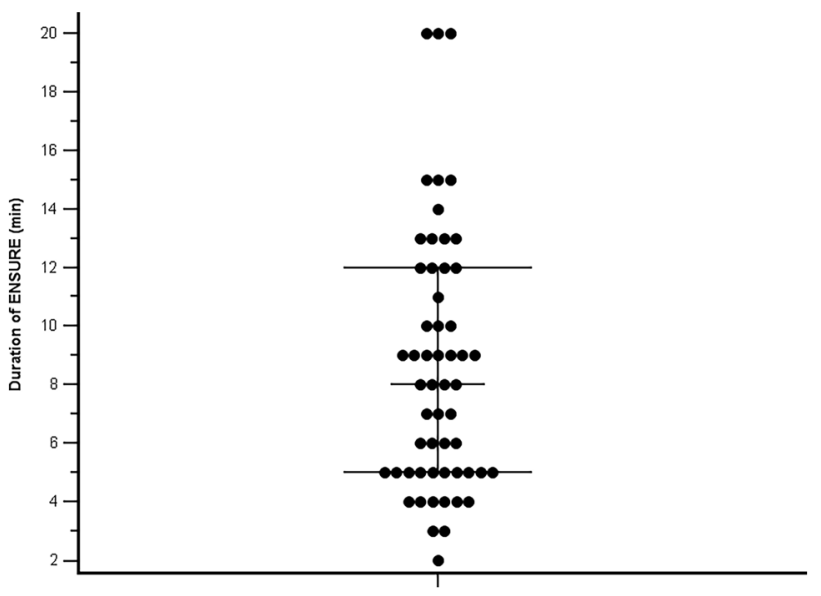

Fig. 2 Duration of enhanced INSURE (ENSURE) procedure in 57 preterm neonates. Horizontal lines indicate median and 25 th -75 th percentiles

and/or much larger volumes and/or much longer ventilation times $[4,5]$. Therefore, in the absence of specific animal data demonstrating that this gentle and short ventilation would be significantly harmful, the risk of ventilator-induced lung injury should be considered very low; further data are needed to estimate it and understand what are the best ventilatory modes and boundaries to be used. As per our respiratory care protocol, we do not guide surfactant administration according to inspired oxygen fraction thresholds: instead, we strive to provide a personalised surfactant therapy. To do so, we use an ultrasound-guided surfactant administration (the so-called ESTHER (echography-guided surfactant therapy)) policy [16]: thus, surfactant was given via ENSURE when lung ultrasound score was $>8$ in neonates of less than 32 weeks' gestation. ENSURE was performed by attending neonatologists or neonatal fellows (with at least 1 year of experience) or residents under the supervision of the former [17]. Residents received at least one low fidelity simulation and were previously briefed on the procedure. ENSURE introduction consisted of the aforementioned standardization and training procedures: the intubation technique itself did not change, nor did the rate of residents' success rate.

\section{Future improvements, limitations and conclusions}

ENSURE represents a formalised way to perform INSURE by updating it to the current respiratory care practice. ENSURE is a peculiar approach to surfactant administration but other techniques might be also effectively used. Nonetheless, ENSURE answers the need to align the technique with current state-of-the-art knowledge and represents an example to standardise INSURE regardless of how it is practiced. 
Thus, ENSURE might reduce variability between teams performing it. In fact, having a poorly defined clinical practice like INSURE can be associated with several drawbacks. First of all, the incertitude about how to perform the technique has somehow led to consider INSURE less suitable and more difficult to realise, compared to other techniques. Second, a poorly defined technique can generate unnoticed and unpredictable effects on clinical outcome and, third, can lower the quality of data when participating to multi-centre studies.

There are still open questions, whose answers would be useful to improve ENSURE further. For instance, the refinement of sedation policy in terms of drug, dose, or timing might be beneficial, as we do not know yet the ideal drug and dose and we cannot recommend the generalised use of one drug over the others. The ideal duration and mode of ventilation should be determined and could be different than those here described. Moreover, the alveolar recruitment before surfactant administration could improve its distribution and biophysical activity, but it is not clear how recruitment should be performed. ENSURE could also be performed with videolaryngoscopy rather than with classical direct laryngoscopy, if specific tools and expertise are available, but this requires dedicated quality improvement projects. Nonetheless, ENSURE yet represents a step forward, as it formally describes an updated technique and can be useful for clinical practice and teaching purposes. It can be applied in all units where expertise, monitoring devices and simulation/teaching facilities would be available. Nonetheless, the feasibility of ENSURE in settings with reduced resource availability might be decreased; however, some tools (such as cerebral saturation or end-tidal $\mathrm{CO}_{2}$ monitoring) are not mandatorily needed or might be replaced by other simpler devices: the feasibility of ENSURE in lowresources setting remains to be determined, and so, it is the best surfactant administration technique.

In conclusion, we offer an updated technique to perform INSURE, clarifying crucial issues of the original technique by applying current state-of-the-art concepts of neonatal respiratory care. ENSURE can be used as a reference technique in clinical practice, simulation teaching and for future studies in the field.

Supplementary information The online version contains supplementary material available at https://doi.org/10.1007/s00431-021-04301-x.

Acknowledgements The authors are grateful to the whole medical and paramedical NICU team for the support in developing ENSURE and the simulation training to implement it.

Authors' contributions Dr. Fortas and Loi drafted the manuscript; contributed to the literature search, data acquisition and interpretation; and took the video; they equally contributed to the manuscript. Dr. Ben-Ammar, Shankar-Aguilera and Yousef contributed to the literature search, data acquisition and interpretation; Dr. Regiroli performed the statistical analysis and contributed to the literature search, data acquisition and interpretation; Prof. De Luca conceptualised the project, contributed to the statistical analysis and supervised the whole project. All authors critically revised the manuscript for important intellectual content and approved the final manuscript as submitted. All authors agreed to be accountable for all aspects of the work in ensuring that questions related to the accuracy or integrity of any part of the work are appropriately investigated and resolved.

Availability of data and materials All data generated or analysed during this study are included in this article and/or its supplementary material files. Further enquiries can be directed to the corresponding author.

Code availability N/A.

\section{Declarations}

Ethics approval Approved by the "Commission d'Ethique de la SRLF"-n.21/33.

Consent to participate Obtained from parents or guardian upon NICU admission.

Consent for publication Obtained from parents of the recorded neonate.

Competing interests Prof. De Luca has received research and educational grants from Chiesi Pharmaceuticals S.p.A. and ABBVIE Inc. He served as lecturer for Airway Therapeutics, Chiesi Pharmaceuticals S.p.A. and ABBVIE Inc. Finally, he has been member of advisory boards for Chiesi Pharmaceuticals S.p.A., ABBVIE Inc. and OPHIREX Inc. He is titular of an equity option of OPHIREX Inc. Dr. Shankar-Aguilera and Yousef received a travel grant from Chiesi Pharmaceuticals S.p.A.. These companies produce surfactants or surfactant proteins, but had no role in design, preparation, review, approval of the manuscript, or decision to submit it for publication. The declared conflicts are all unrelated to the present manuscript. The other authors have no conflicts of interest to disclose.

\section{References}

1. Verder H, Agertoft L, Albertsen P, Christensen N, Curstedt T, Ebbesen F et al (1992) Surfactant treatment of newborn infants with respiratory distress syndrome primarily treated with nasal continuous positive air pressure. A pilot study Ugeskr Laeger 154:2139-2139

2. Patel P, Houck A, Fuentes D (2021) Examining Variations in Surfactant Administration (ENVISION): a neonatology insights pilot project. Children 8(4):261. https://doi.org/10. 3390/children8040261

3. Abdel-Latif ME, Davis PG, Wheeler KI, De Paoli AG, Dargaville PA (2021) Surfactant therapy via thin catheter in preterm infants with or at risk of respiratory distress syndrome. Cochrane Database Syst Rev 5(5):CD011672. https://doi.org/10.1002/14651858. CD011672.pub2

4. De Luca D, Shankar-Aguilera S, Centorrino R, Fortas F, Yousef N, Carnielli VP (2020) Less invasive surfactant administration: a word of caution. Lancet Child Adolesc Health 4(4):331-340. https://doi.org/10.1016/S2352-4642(19)30405-5

5. De Luca D, Shankar-Aguilera S, Bancalari E (2021) LISA/ MIST: Complex clinical problems almost never have easy solutions. Semin Fetal Neonatal Med 26(2):101230. https://doi.org/ 10.1016/j.siny.2021.101230 
6. Vijayasekaran S, Lioy J, Maschhoff K (2016) Airway disorders of the fetus and neonate: an overview. Semin Fetal Neonatal Med 21(4):220-229. https://doi.org/10.1016/j.siny.2016.03.004

7. Moureau R, Yousef N, Poteaux N, De Luca D (2021) Amélioration de la qualité des soins et gestion des risques par la simulation in situ en réanimation néonatale [Quality improvement and risk management using in situ simulation in neonatal intensive care units]. Proceedings of the SoFraSIMS congress Paris [abstract]

8. Topjian AA, Raymond TT, Atkins D, Chan M, Duff JP, Joyner BL et al (2020) Part 4: Pediatric Basic and Advanced Life Support: 2020 American Heart Association Guidelines for Cardiopulmonary Resuscitation and Emergency Cardiovascular Care. Circulation 142(16 suppl 2). https://doi.org/10.1161/ CIR.0000000000000901

9. Smits A, Thewissen L, Caicedo A, Naulaers G, Allegaert K (2016) Propofol dose-finding to reach optimal effect for (semi-)elective intubation in neonates. J Pediatr 179:54-60.e9

10. Thewissen L, Caicedo A, Dereymaeker A, Van Huffel S, Naulaers G, Allegaert K et al (2018) Cerebral autoregulation and activity after propofol for endotracheal intubation in preterm neonates. Pediatr Res 84:719-725. https://doi.org/10.1038/s41390-018-0160-3

11. Singh Y, Tissot C, Fraga MV, Yousef N, Cortes RG, Lopez J et al (2020) International evidence-based guidelines on Point of Care Ultrasound (POCUS) for critically ill neonates and children issued by the POCUS Working Group of the European Society of Paediatric and Neonatal Intensive Care (ESPNIC). Crit Care 24(1):65. https://doi.org/10.1186/s13054-020-2787-9

12. Spence K, Barr P (2000) Nasal versus oral intubation for mechanical ventilation of newborn infants. Cochrane Database Syst Rev 1999(2):CD000948. https://doi.org/10.1002/14651858.CD000948
13. Sweet DG, Carnielli V, Greisen G, Hallman M, Ozek E, te Pas A et al (2019) European Consensus guidelines on the management of respiratory distress syndrome -2019 update. Neonatology 115:432-450

14. Klingenberg C, Wheeler KI, McCallion N, Morley CJ, Davis PG (2017) Volume-targeted versus pressure-limited ventilation in neonates. Cochrane Database Syst Rev 10(10):CD003666. https:// doi.org/10.1002/14651858.CD003666.pub4.

15. Lemyre B, Davis PG, De Paoli AG, Kirpalani H (2017) Nasal intermittent positive pressure ventilation (NIPPV) versus nasal continuous positive airway pressure (NCPAP) for preterm neonates after extubation. Cochrane Database Syst Rev 2(2):CD003212. https://doi.org/10.1002/14651858.CD003212. pub3.

16. Raschetti R, Yousef N, Vigo G, Marseglia G, Centorrino R, BenAmmar R et al (2019) Echography-guided surfactant therapy to improve timeliness of surfactant replacement: a quality improvement project. J Pediatr 212:137-143.e1. https://doi.org/10.1016/j. jpeds.2019.04.020

17. Gomond-LeGoff C, Vivalda L, Foligno S, Loi B, Yousef N, De Luca D (2020) Effect of different probes and expertise on the interpretation reliability of point-of-care lung ultrasound. Chest 157:924-931. https://doi.org/10.1016/j.chest.2019.11.013

Publisher's Note Springer Nature remains neutral with regard to jurisdictional claims in published maps and institutional affiliations. 